\title{
IRT Item Bias Detection Procedures: Issues of Model Misspecification, Robustness, and Parameter Linking
}

\author{
Gary d. Lautenschlager and Dong Gum Prak \\ University of Georgia
}

\begin{abstract}
This article examines the consequences of employing IRT item bias detection procedures with multidimensional IRT item data. Parameter linking methods used in previous studies of item bias were investigated in a simulation that minimized the need for such linking. The results illustrate shortcomings of two linking methods that have been employed in IRT item bias detection studies. The effectiveness of these methods depended on several factors, including the number of biased items in a fixed-length test, whether bias was against only one group or more than one group, and the correlation between the two latent abilities. The findings indicated that some current IRT-based statistical procedures for detecting item bias were not generally effective at differentiating biased from unbiased items. Index terms: item bias, item response theory, multidimensional IRT data, parameter linking, reverse bias, statistical artifacts.
\end{abstract}

In item response theory (IRT), methods used to link $\theta$ metrics seem to be considered unimportant by many researchers, as few published item bias studies have reported which linking procedure was employed to study item bias (Linn, Levine, Hastings, \& Wardrop, 1981; McCauley \& Mendoza, 1985; Shepard, Camilli, \& Williams, 1984, 1985). This tendency not to discuss linking methods probably occurs because researchers believe the procedures are quite straightforward. Researchers have treated the metric linking problems in IRT item bias research as essentially the same as those encountered in metric linking situations involving samples taken from the same population. For example, in

APPLIED PSYCHOLOGICAL MEASUREMENT

Vol. 12, No. 4, December 1988, pp. 365-376

(C) Copyright 1988 Applied Psychological Measurement Inc. 0146-6216/88/040365-12\$1.85 presenting a new linking method, Stocking and Lord (1983) stated:

Suppose that item parameters for a given set of irems, all measuring a single ability or trait, have been independently estimated using data obtained from two different groups of examinees. These item parameter estimates will be different because the metric or scale defined by each independent calibration of the items is different. Many applications of item response theory (IRT) not only require adequate fit of the IRT model to the data but also require that item parameter estimates from independent calibrations be expressed in the same metric. Such applications include vertical scoreequating, horizontal score-equating, and item bias studies. (p. 201)

These procedures are legitimate when used in ordinary metric linking situations in which the samples are assumed to be from the same population. However, if samples come from different populations it is not clear that these methods are appropriate.

Currently, all IRT item bias detection methods compare the item characteristic curves (ICCS) obtained separately from the groups under investigation. Putting item scales and person parameters on the same scale so they can be compared is an important step in item bias detection because the item scale is indeterminate and person parameter estimates are obtained separately for each group. Developing comparable scales involves linking ability metrics through a standardization process that includes item difficulty parameter estimates, 
and a number of methods have been developed that could ostensibly serve this purpose (Divgi, 1985; Linn et al., 1981; Lord, 1980; Stocking \& Lord, 1983; Warm, 1978).

For example, Lord's (1980) parameter estimation procedures, which have been frequently cited as "standard procedure" in the item bias literature, portrayed the metric linking step as technically and conceptually simple. Lord's metric linking procedure identified the following steps:

1. Estimate approximate item parameters for all groups combined, standardizing on $b_{i}$ and not on $\theta$;

2. Fix $c_{i}$ at the values obtained in step $\mathbb{1}$, reestimate $a_{i}$ and $b_{i}$ separately for each group, standardizing on $b_{i}$; and

3. For each item, compare across groups the ICCS of parameters obtained in step 2.

Lord (1980) gave a rationale for standardizing on $b_{i}$. He stated, ". . . standardizing on the $b_{i}$ means that the scale is chosen so that the mean of the $b_{i}$ is 0 and the standard deviation is 1 . Except for sampling fluctuations, this automatically places all parameters for all groups on the same scale. If the usual method of standardizing on $\theta$ were used, the item parameters for each group would be on a different scale"' (p. 217).

Following this basic logic, several researchers have revised the linking procedure to reduce the effects of sampling error (Divgi, 1985; Linn et al., 1981; Lord, 1980; Stocking \& Lord, 1983). Subsequently, researchers investigating IRT-based item bias have used one of these procedures. For example, McCauley and Mendoza (1985) used the simplest procedure described by Warm (1978), whereas Shepard et al. $(1984,1985)$ employed the procedure devised by Linn, Levine, Hastings, and Wardrop (1980).

\section{Conceptural Problems ing

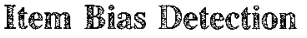

Unfortunately, these procedures have important logical flaws when applied to item bias studies. Two related differences exist between ordinary metric linking situations (e.g., vertical or horizon- tal score equating) and item bias analyses of IRT models. The first difficulty arises when using a unidimensional IRT model in the presence of biased items, when the viability of the unidimensionality assumption is necessarily suspect. The unidimensionality assumption should not be made in item bias studies where an incidental (second) $\theta$ dimension might affect responses to a subset of items. Thus, unidimensionality must be tested-not assumed-in all situations that use a unidimensional IRT model.

This poses a real dilemma when assessing item bias. If unidimensionality is assumed and a unidimensional IRT model (and subsequently a metric linking method) is applied that relies on this assumption to examine item bias, then the degree of bias detected in the process, as estimated by ICCs, may be distorted. The robustness of parameter estimates obtained when fitting multidimensional IRT data using a unidimensional IRT parameter estimation procedure would be an important consideration in such investigations (Ansley \& Forsyth, 1985).

The second problem in IRT item bias analyses is that the linking methods may not apply equally well to all the "common" items for the groups. This contrasts with IRT metric linking in which it is assumed that samples are drawn from the same population, so that the linking methods do apply equally well to the common iterns (Vale, 1986). Although item bias analyses may use exactly the same set of items administered to different groups, this usually violates the assumption that the samples are drawn from the same population. Indeed, the very purpose of IRT item bias analyses-finding different ICCs for the same item-illustrates this point. Thus, the same literal set of items is not always a true "common" set of items in terms of parameter linking.

Obviously, common items in ordinary linking procedures are the items that have identical ICCs (within a linear transformation) for the groups under study. In the IRT framework, if no biased items exist, then parameter linking places the two sets of parameter estimates on a common scale due to item-parameter invariance. However, for biased 
items, item-parameter invariance does not hold; if bias exists, different ICCs exist for the groups on the dimension of interest. Thus, standardizing on $b_{i}$ may not successfully place the groups' parameter estimates onto the same scale (this problem has been recognized by $\operatorname{Linn}$ et al., 1980). In fact, standardizing on $b_{i}$ in the presence of biased items must result in parameter estimates for the groups that are on different scales. The result is that unbiased items appear biased and/or biased items appear unbiased.

These points, taken together, serve as the primary focus of the present study. This study investigated the effectiveness of employing a unidimensional IRT item parameter estimation procedure using multidimensional item data, and then compared the results of two parameter linking methods that have been used in IRT item bias analysis.

\section{Method}

\section{Linghing Methods}

The linking methods employed were Linn et al.'s (1980) and Warm's (1978), and a "no-linking" baseline condition which is described below. Warm's method is the simplest; it is based on the concept that the item difficulty values are identical across groups, within a linear transformation. Warm's method proceeds by standardizing item difficulty parameter estimates $b_{i}$ for one group relative to the values from another group using standard linear linking formulas:

$b_{1}=\frac{\mathrm{SD} b_{1}}{\mathrm{SD} b_{2}} b_{2}+\left(\mathrm{M} b_{1}-\frac{\mathrm{SD} b_{1}}{\mathrm{SD} b_{2}} \mathrm{M} b_{2}\right)$

$a_{1}=a_{2} \frac{\mathrm{SD} b_{2}}{\mathrm{SD} b_{1}}$

where $b_{1}$ is the item $b$ on the metric of group 1 ; $b_{2}$ is the item $b$ on the metric of group 2; $a_{1}$ is the item $a$ on the metric of group 1 ; $a_{2}$ is the item $a$ on the metric of group 2; $\mathrm{M} b_{1}$ and $\mathrm{SD} b_{1}$ are the mean and standard deviation of item $b$ s on the metric of group 1 ; and
$\mathrm{M} b_{2}$ and $\mathrm{SD} b_{2}$ are the mean and standard deviation of the bs on the metric of group 2 .

Limn et al.'s (1980) linking method is similar to Warm's (1978), but it estimates the accuracy of the item difficulty parameters. It substitutes the weighted means and standard deviations for the ordinary means and standard deviations used in Warm's method. These weights are derived separately for each item. A given item's weight is the inverse of the largest estimated sampling variances of the $b_{i}$ values obtained for the $i$ th item, as estimated separately in each group. Thus, items from either group that have relatively larger sampling variances for the difficulty parameter estimates are given less weight in determining parameter linking constants than items with smaller sampling variances. These linking constants cant then transform the $a$ parameter estimates and $\theta$ values to a common scale (Lord, 1980).

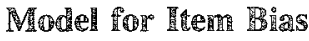

A common type of biasing situation was simulated in which item bias results from multidimensionality which confounds differences on a focal trair with differences on an incidental trait. Specifically, a situation was simulated in which an incidental dimension affected test item performance for one group and not for another. A frequently cited example of items involving both a focal and an incidental dimension is a math test composed of word problems (e.g., Shepard et al., 1984). Although such tests generally are constructed to require only basic reading skills, group differences on the incidental dimension of reading can affect item performance.

Assume one subpopulation in which all examinees comprehend the questions, so that the only trait infuencing test performance is math ability. Suppose that reading ability is lower in a second subpopulation so that not all examinees fully conprehend all questions. In this situation, both math and reading abilities influence item performance. Thus, the items are unidimensional for one group while some of the same items are multidimensional 
in the other group (Hambleton \& Swaminathan, 1985).

Although various operational definitions for simulating item bias have been employed (e.g., Drasgow, 1987; McCauley \& Mendoza, 1985), the present study used an incidental $\theta$ dimension (Park, 1988). It appears to be consistent with the IRT concept of item bias to assume that an incidental dimension could only affect the item responses of one group, as item bias can imply that unidimensional items for one group are multidimensional for another group. Making this assumption also remains conceptually consistent with previous simulation studies of item bias that have simply modified the $b_{i}$ parameters of some items for one group of examinees, though distinctions exist between some methods in which parameters are not interchangeable (Park, 1988). In the present study, this operational definition of item bias permitied equal a distributions on the focal dimension, while allowing a second incidental dimension to influence performance on some items for one group.

The first step in creating a biased item was selecting a multidimensional model. Among the several multidimensional models available, the model proposed by Sympson (1978) was best suited to this situation. The Sympson noncompensatory model is

$$
=c_{j}+\frac{1-c_{j}}{\prod_{h=1}^{n}\left\{1+\exp \left[-1.7 a_{j h}\left(\theta_{i h}-b_{j h}\right)\right]\right\}},
$$

where $\theta_{i h}$ is the ability parameter for person $i$ on dimension $h$,

$a_{j h}$ is the discrimination parameter for item $j$ on dimension $h$,

$b_{j h}$ is the difficulty parameter for item $j$ on dimension $h$, and

$c_{j}$ is the pseudo-guessing parameter for item $j$.

Ansley and Forsyth (1985) justified using Sympson's noncompensatory model rather than other models by noting that its noncompensatory view of dimensionality is more reasonable for most achievement tests. In addition, they found that the model produced simulated data with similar properties to actual achievement test data. A two-dimensional version of Sympson's model was used to generate biased items in this study. For all unbiased items, the unidimensional three-parameter logistic model was used to generate data.

Another variable in the data simulations was the direction of bias. Several researchers (Drasgow, 1987; Lord, 1977; Shepard et al., 1984) have reported finding mixed types of item bias, in which some items were biased against one group and others were biased against a different group. Because the evidence confirms the possibility of mixed forms of bias, two bias situations were simulated: tests with biased items all against one group, and tests in which half of the biased items were biased against one group and the other half were biased against the other group.

\section{Selection of Htem Parameters}

Swaminathan and Gifford (1980) and Ansley and Forsyth (1985) emphasized that data in monte carlo studies should simulate real data as realistically as possible. Following these guidelines, item difficulty parameters associated with the focal dimen$\operatorname{sion} \theta_{1}$ were sampled from a uniform distribution in the interval from -2 to +2 , and item discrimination parameters were sampled from a uniform distribution in the interval from .60 to 2. The pseudoguessing parameters were all set at .20 on the basis of Lord's (1980) statement that the pseudo-guessing parameter values for well-constructed tests will often be much lower than suggested by the classical formula. However, other researchers (Shepard et al., 1985) have observed sizable $c$ parameter values, particularly in relatively easy tests.

In order to generate datasets with biased items, the guidelines employed by Ansley and Forsyth (1985) that pertain to item parameters for an incidental dimension $\theta_{2}$ for two-dimensional IRT data were followed. The $a_{j 2}$ parameter values were centered at about .50 with a value of about .10 for a standard deviation. The $b_{j 2}$ values for the biased items were scaled to have a mean of -1 and a 
standard deviation of .70 . Both sets of item parameters were sampled from uniform distributions.

The rationale for these values was that standardized achievement tests are typically designed to tap one basic dimension. The presence of other dimensions occurs because of the nature of the subject matter or the test items. For example, reading ability is probably a secondary ability dimension on most tests. Because items on these tests are not written primarily to assess reading, they probably do not discriminate highly on that dimension. Ansley and Forsyth (1985) reported that by using similar item parameter guidelines they created items similar to actual achievement test item data.

\section{Da每 Ceneration}

The simulation of item response data for examinees was based on variations on the modified three-parameter logistic IRT model. This model was selected because the three-parameter model provides a realistic reflection of data from standardized achievement tests (Ansley \& Forsyth, 1985).

Datasets were generated to simulate item responses to multiple-choice items with four response options for two groups, Group $A$ and Group $B$. The number of examinees and the lest length were 1,000 and 54, respectively, for all simulated datasets. In a given simulated test, the number of biased items was either 18,28 , or 46 . Although using 46 biased items in a 54 -item test may seem unrealistic, including this level of bias allowed examination of how robust the IRT parameter estimation and the item bias detection procedures were in the presence of pervasive bias. Fourteen pairs of datasets were created based on combinations of the number of biased items, trait score distributions on the first and the second dimensions, the correlation of the latent dimensions, and the direction of bias (see Table 1).

Identical normally distributed $\theta$, vectors with a common mean set to 0 and a common variance of 1 were generated for 1,000 simulated examinees in both Group $\mathrm{A}$ and Group $B$. These two vectors were used throughout the simulations to generate item responses for all conditions investigated. This equivalence of ability on the focal dimension was designed to demonstrate the adverse effect of the linking procedures more clearly because no explicit linking should have been needed. The implicit linking for the "no-linking" conditions involved the identity transformation of estimated $\theta$ metrics. This transformation may be close to optimal for the conditions studied. (The identity transformation would be truly optimal for the equivalent $\theta_{1}$ distribution conditions simulated when all the items fit the same unidimensional model for both groups, i.e., when no biased items are present.) Thus, item bias index values calculated under these no-linking conditions served as a baseline for evaluating results obtained by each of the explicit linking methods. This procedure prevented effects from non-equal $\theta_{1}$ distributions for the two groups from being confounded with those that occur due to the linking procedures alone.

Unidirectional bias conditions. It was assumed for the unidirectional bias conditions that only $\theta_{1}$ influenced performance on all unbiased items. Thus, the modified three-parameter logistic IRT model was used to generate item response data for all items in Group A and for all unbiased items in Group $\mathbb{B}$. The generation of item responses for the biased items was based on the two-dimensional version of Sympson's model. Two types of normal distributions of $\theta_{2}$ were generated for Group $\mathbb{B}$ using a mean of either -.5 or 0 , and a standard deviation of 1 . The correlation between $\theta_{1}$ and $\theta_{2}$ was set to either .60 or .90 in the population. A description of the seven datasets generated to represent the unidirectional bias conditions is presented in Table $\mathbb{1}$ (conditions $\mathbb{1}$ through 7).

Mixed directional bias conditions. In generating test item responses with mixed directional bias, it was assumed that a separate, unrelated (across groups) incidental ability influenced performance on biased items within each group. Consequently, two additional sets of item parameters and two distinct incidental $\theta$ dimensions for biased items were generated in a given mixed bias condition. Items biased against one group were unbiased in the other group. 


\begin{tabular}{|c|c|c|c|c|c|c|}
\hline \multirow{2}{*}{$\begin{array}{l}\text { Bias } \\
\text { Condition } \\
\end{array}$} & \multirow[b]{2}{*}{ Group } & \multirow{2}{*}{$\begin{array}{l}\text { Number of } \\
\text { Unbiased } \\
\text { Items }\end{array}$} & \multirow{2}{*}{$\begin{array}{l}\text { Number of } \\
\text { Biased } \\
\text { Items } \\
\end{array}$} & \multicolumn{2}{|c|}{$\begin{array}{c}\text { Normal } \theta_{2} \\
\text { Discribution }\end{array}$} & \multirow[b]{2}{*}{$r\left(\theta_{2} \theta_{2}\right)$} \\
\hline & & & & M & $\mathrm{SD}$ & \\
\hline \multicolumn{7}{|c|}{ Unidirectional } \\
\hline \multirow[t]{2}{*}{1} & A & 54 & 0 & $\cdots$ & -- & $=$ \\
\hline & $B$ & 36 & 18 & -.5 & 1.0 & .60 \\
\hline \multirow[t]{2}{*}{2} & A & 54 & 0 & -- & $\cdots$ & $\cdots$ \\
\hline & $\mathrm{B}$ & 26 & 28 & -.5 & 1.0 & .60 \\
\hline \multirow[t]{2}{*}{3} & A & 54 & 0 & - & - & $=-$ \\
\hline & $B$ & 8 & 46 & -.5 & 1.0 & .60 \\
\hline \multirow[t]{2}{*}{4} & $A$ & 54 & 0 & - & - & $=$ \\
\hline & $B$ & 36 & 18 & -.5 & 1.0 & .90 \\
\hline \multirow[t]{2}{*}{5} & A & 54 & 0 & - - & - & $\cdots$ \\
\hline & 8 & 8 & 46 & -.5 & 1.0 & .90 \\
\hline \multirow[t]{2}{*}{6} & A & 54 & 0 & $\cdots$ & $\cdots$ & $\cdots$ \\
\hline & B & 36 & 18 & 0.0 & 1.0 & .90 \\
\hline \multirow[t]{2}{*}{7} & $A$ & 54 & 0 & $\cdots$ & - & $\cdots$ \\
\hline & B & 8 & 46 & 0.0 & 1.0 & .90 \\
\hline \multicolumn{7}{|c|}{ Mixed Directional } \\
\hline \multirow[t]{2}{*}{8} & A & 45 & 9 & 0.0 & 1.0 & .60 \\
\hline & $B$ & 45 & 9 & -.5 & 1.0 & .60 \\
\hline \multirow[t]{2}{*}{9} & A & 40 & 14 & 0.0 & 1.0 & .60 \\
\hline & $\mathbb{B}$ & 40 & 14 & -.5 & 1.0 & .60 \\
\hline \multirow[t]{2}{*}{10} & A & 31 & 23 & 0.0 & 1.0 & .60 \\
\hline & $\mathbb{B}$ & 31 & 23 & -.5 & 1.0 & .60 \\
\hline \multirow[t]{2}{*}{11} & A & 45 & 9 & -.5 & 1.0 & .90 \\
\hline & $B$ & 45 & 9 & -.5 & 1.0 & .90 \\
\hline \multirow[t]{2}{*}{12} & A & 31 & 23 & -.5 & 1.0 & .90 \\
\hline & $B$ & 31 & 23 & -.5 & 1.0 & .90 \\
\hline \multirow[t]{2}{*}{13} & A & 45 & 9 & 0.0 & 1.0 & .90 \\
\hline & $B$ & 45 & 9 & 0.0 & 1.0 & .90 \\
\hline \multirow[t]{2}{*}{14} & A & 31 & 23 & 0.0 & 1.0 & .90 \\
\hline & $B$ & 31 & 23 & 0.0 & 1.0 & .90 \\
\hline
\end{tabular}

The generation of biased items proceeded as in the unidirectional bias conditions, except that biased items were created for both groups. Again two types of normal $\theta_{2}$ distributions were generated using a mean of either 0 or -.5 with a standard deviation of 1 . The correlation of $\theta_{1}$ with $\theta_{2}$ in the population was set at either.60 or .90 and was the same for both groups within a given bias condition (see conditions 8 through 14 in Table 1).

Creating item responses. The generation of item responses for each of the unbiased items involved only the item parameters for the $\theta_{1}$ vectors for each group, as described earlier. The three-parameter logistic model was used to create the probability of a correct response for a given examinee's $\theta_{1}$ value on a given item. As each probability of a correct response (for a given examinee on a given item) was generated, it was compared with a value sampled from a uniform random number generator in the range from 0 to 1 . If the probability based on the item parameters and the specific $\theta$ value was equal to or exceeded the random number, a value of 1 was recorded for that examinee on that item; otherwise the item was given a 0 . This was done for all unbiased items regardless of bias condition.

The generation of all biased item responses was equivalent to the procedure described in Ansley and Forsyth (1985), using a two-dimensional ver- 
sion of Sympson's (1978) model. The respective item parameters relevant to the two $\theta$ dimensions were paired with the appropriate $\theta$ vector values 10 produce the probabilities of a correct response in the two-dimensional model, which were converted to 0,1 item response values by the procedure described above.

\section{Aralysis}

The datasets were analyzed after using the LOGIST computer program (Wingersky, Barton, \& Lord, 1982) to estimate the item and person parameters separately for each group. After item and person parameter estimates were obtained, the two linking procedures were used to place the parameter estimates obtained separately for each group on the same scale. Then, Lord's (1980) chi-square item bias statistic was calculated for each item under each linking condition. Lord's index was selected because it has a stronger statisîical justification, and it is the only index with an associated significance test. The .005 significance level was used throughout for indicating "detected" item bias.

Lord's (1980) chi-square item bias indices were computed after applying the two item-parameter linking procedures for each item bias condition. In addition, chi-square indices were obtained without explicit parameter linking, to provide a "baseline" for the bias index values.

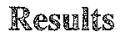

The datasets for Group A and Group B in each bias condition were generated using unit normal distributions of ability values on the focal dimension $\left(\theta_{1}\right)$, resulting in nearly perfect classification of the items as biased or unbiased as might be expected without parameter linking (see Table 2). Indeed, the itenns created as unbiased were almost perfectly classified as unbiased in all of the test bias conditions studied when no linking was used. However, some items created as biased in the population were not detected as biased when no explicit linking was employed. These items may have been only weakly biased; the incidental dimension may have had little effect due to higher difficulty parameter values on the focal dimension already set for these items.

\section{Unilidirectional}

Table 2 presents the number of false positives (FP), false negatives (FN), and correct identifications (HITS) of biased or unbiased itemes in the unidirectional test bias conditions. All of the items created as biased which were subsequently identified as HITs were biased in the intended direction, namely against Group B. However, all FP identifications resulted in detecting reverse bias (against Group A).

For bias conditions 1 through $3\left[\theta_{2} \sim \mathbb{N}(-.5,1)\right.$ and $\left.r\left(\theta_{1}, \theta_{2}\right)=.6\right]$, the proportion of unbiased items identified as FPs tended to increase as the number of biased items increased. A similar tendency occurred for the proportion of biased items identified as FNs to increase for the Limn et al. (1980) and the no-linking condition, but a slight reverse trend existed in Warm's (1978) linking method. Warm's method consistently performed worse with respect to FPS than FNs, for which results were mixed. Linn et al.'s method did better with fewer biased items and Warm's method did better with more biased items. As anticipated, both linking methods performed consistently worse than assuming the identity transformation (e.g., simply not applying a linking method). In addition, as the number of biased items increased, so did the proportion of FNs relative to the number of biased items, even when no linking was done.

For bias conditions 4 and $5\left[\theta_{2} \sim \mathbb{N}(-.5,1)\right.$ and $r\left(\theta_{1}, \theta_{2}\right)=.901$, the results were similar to those in which the correlation between the focal and incidental traits was lower. However, the FP rates appear lower for the 18-biased-item condition when $r\left(\theta_{1}, \theta_{2}\right)$ was .90 than it was in bias condition 1 . Again, as the number of biased items increased the proportion of FNS relative to the number of biased items rose when no linking was used.

Bias condition $6 \mathbb{1} \theta_{2} \sim \mathbb{N}(0,1)$ and $r\left(\theta_{1}, \theta_{2}\right)=$ $.90]$ reveals a tradeoff for the 18 -biased-item condition. A large reduction in the number of FPS oc- 
Table 2

Number of False Positives (FP), False

Negatives (FN), and Correct Identifications (HIT)

in the Unidirectional and Mixed Directional

Iten Bias Conditions

\begin{tabular}{|c|c|c|c|c|c|}
\hline \multirow{2}{*}{$\begin{array}{l}\text { Bias } \\
\text { Condition }\end{array}$} & \multirow{2}{*}{$\begin{array}{l}\text { Linking } \\
\text { Method }\end{array}$} & \multicolumn{2}{|c|}{ Unbiased Items } & \multicolumn{2}{|c|}{ Biased Items } \\
\hline & & $\mathrm{FP}$ & $\mathrm{HIT}$ & FN & $\mathrm{HIT}$ \\
\hline \multicolumn{6}{|c|}{ Unidirectional } \\
\hline \multirow[t]{3}{*}{1} & Linn & 5 & 31 & 3 & 15 \\
\hline & Warm & 19 & 17 & 6 & 12 \\
\hline & None & 2 & 34 & 2 & 16 \\
\hline \multirow[t]{3}{*}{2} & Linn & 7 & 19 & 8 & 20 \\
\hline & Warm & 24 & 2 & 8 & 20 \\
\hline & None & 0 & 26 & 5 & 23 \\
\hline \multirow[t]{3}{*}{3} & Linn & 8 & 0 & 16 & 30 \\
\hline & Wara & 8 & 0 & 12 & 34 \\
\hline & None & 0 & 8 & 11 & 35 \\
\hline \multirow[t]{3}{*}{4} & Linn & 2 & 34 & 3 & 15 \\
\hline & Warm & 15 & 21 & 6 & 12 \\
\hline & None & 0 & 36 & 3 & 15 \\
\hline \multirow[t]{3}{*}{5} & Linn & 8 & 0 & 15 & 31 \\
\hline & Warm & 8 & 0 & 14 & 32 \\
\hline & None & 0 & 8 & 10 & 36 \\
\hline \multirow[t]{3}{*}{6} & Linn & 3 & 33 & 7 & 11 \\
\hline & Warm & 8 & 28 & 8 & 10 \\
\hline & None & $\mathbb{1}$ & 35 & 7 & 11 \\
\hline \multirow[t]{3}{*}{7} & Linn & 4 & 4 & 16 & 30 \\
\hline & Varm & 7 & 1 & 14 & 32 \\
\hline & None & 0 & 8 & 15 & 31 \\
\hline \multicolumn{6}{|c|}{ Mixed Directional } \\
\hline \multirow[t]{3}{*}{8} & Linn & 0 & 36 & 4 & 14 \\
\hline & Warm & 0 & 36 & 4 & 14 \\
\hline & None & 0 & 36 & 3 & 15 \\
\hline \multirow[t]{3}{*}{9} & Linn & 0 & 26 & 5 & 23 \\
\hline & Warm & 0 & 26 & 6 & 22 \\
\hline & None & 0 & 26 & 5 & 23 \\
\hline \multirow[t]{3}{*}{10} & Linn & 0 & 8 & 8 & 38 \\
\hline & Warm & 0 & 8 & 5 & 41 \\
\hline & None & 0 & 8 & 6 & 40 \\
\hline \multirow[t]{3}{*}{11} & Linn & 0 & 36 & 1 & 17 \\
\hline & Warm & 0 & 36 & 1 & 17 \\
\hline & None & 0 & 36 & 1 & 17 \\
\hline \multirow[t]{3}{*}{12} & Limn & 0 & 8 & 9 & 37 \\
\hline & Warm & 1 & 7 & 7 & 39 \\
\hline & None & 0 & 8 & 9 & 37 \\
\hline \multirow[t]{3}{*}{13} & Linn & 0 & 36 & 7 & 11 \\
\hline & Warm & 0 & 36 & 7 & 11 \\
\hline & None & 0 & 36 & 4 & 14 \\
\hline \multirow[t]{3}{*}{14} & Linn & 0 & 8 & 12 & 34 \\
\hline & Warm & 0 & 8 & 12 & 34 \\
\hline & None & 0 & 8 & 14 & 32 \\
\hline
\end{tabular}

Downloaded from the Digital Conservancy at the University of Minnesota, http://purl.umn.edu/93227. May be reproduced with no cost by students and faculty for academic use. Non-academic reproduction requires payment of royalties through the Copyright Clearance Center, http://www.copyright.com/ 
curred when Warm's method was used, but an increase in the proportion of FNS occurred regardless of linking method. When the number of biased items increased to 46 (bias condition 7), Limn's linking method fared better than Warm's with respect to FPS. These last two conditions produced the highest rates of $\mathbb{F N}$ item misclassifications for the unidirectional bias cases, regardless of linking method.

The results for bias conditions 3,5 , and 7 , in which the amount of bias was clearly pervasive ( 46 biased items), showed that the unbiased items were correctly identified when no explicit linking was employed. These results suggest that LOGIST was able to produce reasonable item parameter estimates despite the pervasive bias, and that the unidimensional estimation procedure was robust to the multidimensionality present in the biased items. Apparently, the major factor contributing to false positive item misclassifications for the Warm (1978) and Linn et al. (1980) linking conditions was the linking method itself. The fitting of the unidimensional IRT model to the datasets with a large number of biased (multidimensional) items appeared to make some multidimensional items appear more like unidimensional items (i.e., produce false negative misclassifications of biased items).

Table 3 presents information about the extent to which false negatives were identified by each linking method. The maximum amount of agreement in any given column is determined by the minimum number of FNs among the methods being compared. For example, in bias condition 1 the maximum possible number of common FNs is two for

Table 3

Number (N) and Maxinum Possible Number (M) of False

Negatives (FN) Common and Unique to Linking Methods Identified in the Unidirectional and Mixed Directional Item Bias Conditions

\begin{tabular}{|c|c|c|c|c|c|c|c|c|c|c|c|c|}
\hline \multirow{3}{*}{\multicolumn{2}{|c|}{$\begin{array}{l}\text { Bias } \\
\text { Condition }\end{array}$}} & \multicolumn{8}{|c|}{ Common ENs } & & & \\
\hline & & \multicolumn{2}{|c|}{$\begin{array}{c}\text { All } \\
\text { Methods }\end{array}$} & \multicolumn{2}{|c|}{$\begin{array}{l}\text { Linn } \\
\& \text { None }\end{array}$} & \multicolumn{2}{|c|}{$\begin{array}{c}\text { Warm } \\
\& \text { None }\end{array}$} & \multicolumn{2}{|c|}{$\begin{array}{c}\text { Linn } \\
\text { \& Warm }\end{array}$} & \multicolumn{3}{|c|}{ Unique FNs } \\
\hline & & $\mathrm{N}$ & $\mathrm{M}$ & $\mathrm{N}$ & $M$ & $\mathrm{~N}$ & $\bar{M}$ & $\bar{N}$ & $\mathrm{M}$ & Linn & Warm & None \\
\hline \multicolumn{13}{|c|}{ Unidirectional } \\
\hline 1 & & 2 & 2 & 2 & 2 & 2 & 2 & 3 & 3 & 0 & 3 & 0 \\
\hline 2 & & 5 & 5 & 5 & 5 & 5 & 5 & 8 & 8 & 0 & 0 & 0 \\
\hline 3 & & 4 & 11 & 10 & 11 & 4 & 11 & 10 & 12 & 0 & 2 & 1 \\
\hline 4 & & 3 & 3 & 3 & 3 & 3 & 3 & 3 & 3 & 0 & 3 & 0 \\
\hline 5 & & 6 & 10 & 9 & 10 & 6 & 10 & 10 & 14 & 2 & 4 & 1 \\
\hline 6 & & 7 & 7 & 7 & 7 & 7 & 7 & 7 & 7 & 0 & 1 & 0 \\
\hline 7 & & 9 & 14 & 12 & 15 & 9 & 14 & 12 & 14 & 1 & 2 & 3 \\
\hline \multicolumn{13}{|c|}{ Mixed Directional } \\
\hline \multirow[t]{2}{*}{8} & A & 1 & 1. & 1 & 1 & 1 & 1 & 1 & 1 & 0 & 0 & 1 \\
\hline & B & 1 & 1 & 1 & 1 & 1 & 1 & 3 & 3 & 0 & 0 & 0 \\
\hline \multirow[t]{2}{*}{9} & A & 2 & 2 & 3 & 3 & 2 & 2 & 2 & 2 & 0 & 0 & 0 \\
\hline & $B$ & 2 & 2 & 2 & 2 & 2 & 2 & 2 & 2 & 0 & 2 & 0 \\
\hline \multirow[t]{2}{*}{10} & $A$ & 3 & 3 & 3 & 3 & 2 & 3 & 2 & 3 & 0 & 1 & 0 \\
\hline & B & 1 & 1 & 3 & 3 & 1 & $I$ & 1 & 1 & 2 & 0 & 0 \\
\hline \multirow[t]{2}{*}{11} & A & 0 & 0 & 0 & 0 & 0 & 0 & 0 & 0 & 0 & 0 & 0 \\
\hline & B & 1 & 1 & 1 & 1 & 1 & 1 & 1 & 1 & 0 & 0 & 0 \\
\hline \multirow[t]{2}{*}{12} & A & 3 & 3 & 3 & 3 & 3 & 3 & 3 & 3 & 0 & 3 & 0 \\
\hline & $B$ & 1 & 1 & 6 & 6 & 1 & 1 & 1 & 1 & 0 & 0 & 0 \\
\hline \multirow[t]{2}{*}{13} & $A$ & 2 & 2 & 2 & 2 & 2 & 2 & 2 & 2 & 0 & 0 & 0 \\
\hline & B & 2 & 2 & 2 & 2 & 2 & 2 & 5 & 5 & 0 & 0 & 0 \\
\hline \multirow[t]{2}{*}{14} & $\mathrm{~A}$ & 6 & 6 & 6 & 6 & 6 & 6 & 6 & 6 & 0 & 0 & 0 \\
\hline & B & 5 & 6 & 6 & 6 & 6 & 6 & 5 & 6 & 0 & 0 & 1 \\
\hline
\end{tabular}

Downloaded from the Digital Conservancy at the University of Minnesota, http://purl.umn.edu/93227. May be reproduced with no cost by students and faculty for academic use. Non-academic reproduction requires payment of royalties through the Copyright Clearance Center, http://www.copyright.com/ 
the "All Methods" column. Clearly, the "Linn \& Warm" column shows that these two linking methods overlap most in terms of identifying items created to be biased as being unbiased. Warm's method is perhaps more extreme in terms of unique identifications of FNs, whereas Linn's method produces the least unique identifications. This latter finding may be attributed to Linn's method having produced FNS that variously overlap with FNs due to use of either Warm's method or not explicitly linking.

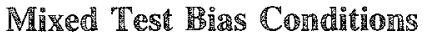

The results from the mixed test bias conditions, in which half of the biased items were biased against Group $A$ and half of them against Group B, are presented in Table 2. In all cases, items created as biased and identified as Hirs were biased in the intended direction. One striking finding that immediately sets these results apart from the unidirectional bias results was the virtual absence (with a single exception) of FP item misclassifications regardless of the bias condition simulated or the linking method employed.

The highest proportion of FNs relative to the total number of biased items occurred in bias conditions 13 and 14 , in which the means of the $\theta_{2}$ dimensions for Group $A$ and Group $B$ were both 0 and the correlations between each $\theta_{2}$ dimension and the focal dimension were both 90 . For bias conditions in which the $\theta_{2}$ dimensions had equivalent means for the two groups (bias conditions 11 through 14), an increase in the number of FNS occurred as the means shifted from -.5 to 0 in both the 18- and 46-biased-item conditions. For the mixed bias conditions simulated in the present study, it appeared that using no explicit linking was roughly the same as each linking method. Under some conditions, either the Linn et al. (1980) and/or the Warm (1978) linking procedure actually performed slightly better than not explicitly linking.

Table 3 presents the common and unique aspects of false negative item identifications by each linking method employed. As in the unidirectional bias conditions, Warm's method produced more unique FN identifications than the other methods.

\section{Discussion}

This study showed that item parameter linking in a multidimensional context is not the same as that involved for samples presumed to come from the same population. The item parameter invariance property in IRT is valid only for unbiased items; therefore, not all test items may be considered "common" items. The results indicate that the item parameter linking methods were adversely affected by the presence of biased items, and often produced inadequate linking results in the form of item misclassifications.

Using a unidimensional IRT item parameter estimation procedure with multidimensional IRT items in this simulated example indicated that the item parameters for truly unbiased items do not get substantially distorted (or at least they are not identified as biased). This finding is supported by the fact that conditions that involved no explicit linking resulted in almost perfect classification of unbiased items as unbiased; this was true even when dealing with large numbers of biased items. This may be a positive finding for item bias researchers, especially because practical multidimensional IRT parameter estimation procedures are not readily available. However, the simulated data were based on a common true $\theta_{1}$ dimension which would never be known, nor safely assumed, in practice.

In the unidirectional bias conditions, Warm's (1978) linking method seemed more affected by the estimated parameters of the biased items, and became progressively more ineffective as the proportion of biased items increased. The present results of high FP rates associated with Warm's linking method are consistent with results reported by McCauley and Mendoza (1985, p. 395) for the chisquare index of item bias. The Linn et al. (1980) method generally did as well as-or better thanWarm's method for the unidirectional bias conditions.

Finding relatively high FP rates in the unidirectional bias conditions is somewhat problematic. Because "biased" items as identified here were biased against Group $A$ (indicating reverse bias), it seems reasonable that other conditions could produce mixed forms of detected item bias when only 
unidirectional bias exists. For example, in unidirectional bias condition 1 in Table $\mathbb{1}$, one-third of the items were created to be biased against Group B. Roughly one-third of the items were subsequently detected as biased, but approximately $10 \%$ of the items were FP identifications (using Linn's linking method), and all of these indicated reverse bias.

Comparing this result with actual results obtained by Shepard et al. (1984, p. 104) is interesting. They detected about one-third of the math word problems as biased, and approximately $10 \%$ of the math items they investigated (using IRT methods with Linn's linking method) were biased in favor of blacks. Perhaps this is just a coincidence. Nonetheless, it is interesting in light of several IRT item bias studies involving actual test data that have detected mixed forms of item bias (e.g. . Drasgow, 1987; Lord, 1977; Shepard et al., 1984).

When true bias was mixed, both linking methods almost perfectly identified unbiased items, perhaps because the scale distortion for the two groups was nearly identical and may have cancelled some distortion. Because true item bias for any given test is unknown, the results obtained in the mixed bias conditions cannot justify using either Warm's or Linn et al.'s linking methods. In addition, the FN rates in the mixed bias conditions were nearly as large as for the unidirectional bias conditions.

The high $\mathrm{FN}$ rates observed under various conditions are also troublesome. Perhaps some biased items are weakly biased, and therefore not always detected as biased. Another reason for the high $\mathrm{FN}$ rate was using a small $p$ value for the significance tests. If a less restrictive probability value were used, fewer FNS would be identified, but FPS would increase. Such tradeoffs must be carefully considered. The FN rates observed appear higher than expected if this were the only factor involved. Given the large proportion of items created as biased under some conditions, some $\theta$ scale distortion probably occurred, which caused the parameter estimation procedure itself to contribute to the FN rate.

Ansley and Forsyth (1985) reported such distortion when investigating the effects of using a unidimensional IRT model with multidimensional data. In fact, even when the correlation was .95 between the focal and incidental traits, appreciable $\theta$ scale distortion still existed. An increasing FN rate as the number of biased items in the test rose is consistent with this explanation. However, the truly unbiased items were not distorted or detected as biased when no explicit linking was employed, even when large numbers of biased items were present.

\section{Conchlusions}

These results demonstrate that under the unidirectional bias circumstances simulated, it was possible to detect a considerable number of reversed bias items that were largely due to the item parameter linking method employed. This makes selecting a metric linking method for IRT item bias research quite difficult. Given the differences in the results for the two linking methods employed here, researchers must begin to report the item parameter linking method used in IRT item bias studies. In practice, it is not easy to justify assuming equal $\theta$ distributions on the focal dimension (though it might be interesting to entertain the consequences of that possibility) and thus a clear need exists for some way to link parameter estimates. In selecting between the two linking methods used in this study, preference might be given to the Linn et al. (1980) method. However, caution is advised in extrapolating the results obtained here under simulated conditions created to minimize the need for linking item parameter metrics to the more uncertain contexts involving actual test data.

The present results further support the notion that the statistical indication of item bias must be followed by examining item content/context (or even sample characteristics) to determine whether identifying the item as biased would be justifiable. The fact that previous item bias research involving actual test data has been largely unable to disentangle the nature of reverse bias items, as identified by item bias indices, makes the results reported here even more compelling. Additionally, false negatives may pose a real problem, as items identified as unbiased by IRT item bias methods may truly be biased. 
The findings reported here are limited to noniterative methods of linking as applied to multidimensional IRT data. Such non-iterative linking methods may not be very useful in IRT item bias studies. Since this study was conducted, Drasgow (1987) has presented an iterative procedure for linking metrics and reported considerable success in correctly identifying simulated items as biased or unbiased. Perhaps such iterative procedures can aid in identifying the truly "common" items to determine the linking constants, in the sense discussed earlier (Vale, 1986). However, Drasgow (1987) simulated biased item responses in a manner quite different from that used in the present study. Regardless of these differences, such iterative procedures may prove useful in light of multidimensional IRT data on which IRT item bias analyses are based (Park, 1988). Clearly an important focus for future IRT item bias research should be on improving item bias detection methods in the presence of multidimensional IRT data.

\section{紫eferences}

Ansley, T. N., \& Forsyth, R. A. (1985). An examination of the characteristics of unidimensional IRT parameter estimates derived from two-dimensional data. Applied Psychological Measurement, 9, 37-48.

Divgi, D. R. (1985). A minimum chi-square method for developing a common metric in item response theory. Applied Psychological Measurement, 9, 413-415.

Drasgow, F. (1987). Study of the measurement bias of two standardized psychological tests. Sournal of Applied Psychology, 72, 19-29.

Hambleton, R. K., \& Swaminathan, H. (1985). Item response theory: Principles and applications. Hingham MA: Kluwer-Nijhoff.

Linn, R. L., Levine, M. V., Hastings, C. N., \& Wardrop, J. L. (1980). An investigation of item bias in a test of reading comprehension (Tech. Rep. No. 163). Urbana IL: University of Illinois, Center for the Study of Reading.

Linn, R. L., Levine, M. V., Hastings, C. N., \& Wardrop, J. L. (1981). Item bias in a test of reading comprehension. Applied Psychological Measurement, 5, $159-163$.

Lord, F. M. (1977). A study of item bias, using item characteristic curve theory. In Y. H. Poortinga (Ed.), Basic problems in cross-cultural psychology (pp. 1929). Amsterdam: Swets \& Zeitlinger.

Lord, F. M. (1980). Applications of item response theory to practical testing problems. Hillsdale NI: Erlbaum.
McCauley, C. D., \& Mendoza, J. (1985). A simulation study of item bias using a two-parameter irem response model. Applied Psychological Measurement, 9, 389400.

Park, D. G. (1988). Investigations of item response theory item bias detection. Unpublished doctoral dissertation, University of Georgia.

Shepard, L. A., Camilli, G., \& Williams, D. M. (1984). Accounting for statistical artifacts in item bias research. Journal of Educational Statistics, 9, 93-128.

Shepard, L. A., Camilli, G., \& Williams, D. M. (1985). Validity of approximation techniques for detecting item bias. Journal of Educational Measurement, 22, 77105

Stocking, M. L., \& Lord, F. M. (1983). Developing a common metric in item response theory. Applied Psychological Measurement, 7, 201-210.

Swaminathan, H., \& Gifford, J. A. (1980). Estimation of parameters in the three parameter latent trait model (Rep. No. 90). Amherst MA: University of Massachusetts, School of Education, Laboratory of Psychometric and Evaluation Research.

Sympson, I. B. (1978). A model for testing with multidimensional items. In D. J. Weiss (Ed.), proceedings of the 1977 Computerized Adaptive Testing Conference (pp. 82-98). Minneapolis: University of Minnesota, Department of Psychology, Psychometric Methods Program.

Vale, C. D. (1986). Linking item parameters onto a common scale. Applied Psychological Measurement, $10,333-344$.

Warm, T. A. (1978). A primer of item response theory (Tech. Rep. No. 941078). Washington DC: U.S. Coast Guard Institute.

Wingersky, M. S., Barton, M. A., \& Lord, F. M. (1982). LOGIST user's guide. Princeton NJ: Educational Testing Service.

\section{Ado}

The authors are listed alphabetically, and contributed equally to the ideas presented. This article is based in part on data from the second atuthor's doctoral dissertation completed at the University of Georgia. The authors thank two anonymous reviewers and the Editor for their helpful comments.

\section{Aanthorrs Address}

Send requests for reprints or further information to either Gary $\mathbb{I}$. Lautenschlager, Depariment of Psychology, University of Georgia, Athens GA 30602, U.S.A. or to Dong-Gun Park, 465-8 Sokyo-Dong, Mapo-Gu, Seoul, Korea. 